\title{
CHAPMAN-ENSKOG-HILBERT EXPANSION FOR THE ORNSTEIN-UHLENBECK PROCESS AND THE APPROXIMATION OF BROWNIAN MOTION
}

\author{
BY \\ RICHARD S. ELLIS(1)
}

\begin{abstract}
Let $(x(t), v(t))$ denote the joint Ornstein-Uhlenbeck position-velocity process. Special solutions of the backward equation of this process are studied by a technique used in statistical mechanics. This leads to a new proof of the fact that, as $\epsilon \downarrow 0, \epsilon x\left(t / \epsilon^{2}\right)$ tends weakly to Brownian motion. The same problem is then considered for $v(t)$ belonging to a large class of diffusion processes.
\end{abstract}

1. Introduction. Previous work on the Boltzmann equation has motivated the following problem. For $\epsilon>0$ a real parameter, let $f=f(x, v), x$ and $v$ real, be a formal power series in $\epsilon$ of the form $\Sigma_{j \geqslant 0} f_{j} \epsilon^{j}$, where the coefficients $f_{j}$ are $C^{\infty}$ functions of $x$. We seek solutions $p=p_{\epsilon}(t, x, v), t>0$, of the equation

$$
\frac{\partial p}{\partial t}+v \frac{\partial p}{\partial x}=\frac{1}{\epsilon} Q p, \quad \lim _{t \downarrow 0} p=f
$$

which are also formal power series in $\epsilon$. In (1.1), $Q$ is a negative semidefinite operator on some Hilbert space which operates on $p$ as a function of $v$ only. Equation (1.1) has the form of a model Boltzmann equation. The study of the existence and properties of such special solutions is known as the Chapman-EnskogHilbert expansion for (1.1).

In $\S 2$ of this paper, we consider this problem for the case when $Q$ is the infinitesimal generator of the Ornstein-Uhlenbeck velocity process $\{v(t) ; t \geqslant 0\}$. If we let $v \rightarrow-v$ in (1.1), then the resulting equation is the backward equation of the joint process $\{x+\epsilon x(t / \epsilon), v(t / \epsilon)\}$, where $x$ is real and $x(t)$ denotes the

Presented to the Society, January 14, 1974; received by the editors June 14,1973 and, in revised form, September 18, 1973.

AMS (MOS) subject classifications (1970). Primary 60J60, 60F05, 35Q99.

Key words and phrases. Chapman-Enskog-Hilbert expansion, Ornstein-Uhlenbeck process, Brownian motion, essentially selfadjoint operator, weak solution of a partial differential equation, diffusion process.

(1) This research was supported in part by National Science Foundation Grant GP-28576. 
position of a particle at time $t$ when its velocity is $v(t)$ and $x(0)=0$. Theorem 2.2 states the main properties of formal power series solutions of this equation. We call the reader's attention to the simple formula (2.6) which expresses in closed form the special power series solutions of our equation. The question of convergence of these formal solutions is also taken up. In $\S 3$, we use these results to give a new proof of the fact that as $\epsilon \downarrow 0$ the scaled process $\epsilon x\left(t / \epsilon^{2}\right)$ converges weakly to a standard Brownian motion. Finally, in $\S 4$, we single out a class of multidimensional diffusion processes for which the formal power series of $\$ 2$ can be carried out.

The spirit of $\S 2$ of this paper is similar to that of [2], in which $Q$ was the infinitesimal generator of a Markov chain with finite state space. For the proof of limit theorems for the situation treated in [2], see [3].

Acknowledgement. The author wishes to thank the referee for several helpful remarks.

2. Chapman-Enskog-Hilbert expansion for the Ornstein-Uhlenbeck process. Let $\{w(t) ; t \geqslant 0\}$ be a standard Brownian motion. We consider the joint Gaussian-Markov process $\left\{\left(x_{y}(t), v(t)\right) ; t \geqslant 0\right\}$ defined by the pair of stochastic differential equations

$$
\begin{aligned}
d x_{y}(t) & =v(t) d t, & d v(t) & =-v(t) d t+d w(t), \\
x_{y}(0) & =y, & v(0) & =v,
\end{aligned}
$$

where $y$ and $v$ are fixed real numbers. The first component $x_{y}(t)$ is known as the Ornstein-Uhlenbeck (position) process. We denote by $P_{(y, v)}\{\cdot\}$ the probability law for the joint process $\left(x_{y}(t), v(t)\right)$ and by $E_{(y, v)}\{\cdot\}$ integration with respect to this measure. For $f=f(y, v)$ suitably smooth, one shows that the function $p(t, y, v)=E_{(y, v)}\left\{f\left(x_{y}(t), v(t)\right)\right\}$ satisfies the partial differential equation

$$
\frac{\partial p}{\partial t}=v \frac{\partial p}{\partial y}+\frac{1}{2} \frac{\partial^{2} p}{\partial v^{2}}-v \frac{\partial p}{\partial v}, \quad \lim _{t \downarrow 0} p=f
$$

The main facts about the operator $Q=1 / 2 \partial^{2} / \partial v^{2}-v \partial / \partial v$ are stated in the next lemma.

Lemma 2.1. Let $H$ denote the Hilbert space $L^{2}(m)$, where $m(d v)$ is the probability measure $\pi^{-1 / 2} \exp \left(-v^{2}\right) d v$. Let $D=\left\{g \in H: g \in C^{2}, Q g \in H\right\}$. Then $Q$ on $D$ is essentially selfadjoint. The Hermite polynomials $\left\{h_{n}(v) ; n \geqslant 0\right\}$ satisfy $Q h_{n}=-n h_{n}$ and have as generating function

$$
\sum_{n \geqslant 0} h_{n}(v) \frac{x^{n}}{n !}=\exp \left(2 x v-x^{2}\right)
$$


the functions $\left\{\left(2^{n} n !\right)^{-1} h_{n}(v)\right\}$ form an orthonormal basis of $H$. If $\bar{Q}$ denotes the selfadjoint closure of $Q$, then the spectra and the eigenfunctions of $Q$ and $\bar{Q}$ coincide.

Proof. Since $Q$ can be written

$$
\frac{1}{2} \exp \left(v^{2}\right) \frac{\partial}{\partial v}\left(\exp \left(-v^{2}\right) \frac{\partial}{\partial v}\right)
$$

one has by $[4, \mathrm{pp} .86,189]$ that $Q$ on $D$ is essentially selfadjoint. The facts about the Hermite polynomials are proven in [1, pp. 91-93]. The last statement in the theorem follows from $[4$, p. 190].

Writing $\exp (t Q)$ for the contraction semigroup on $H$ generated by $Q$, one can show by means of Lemma 2.1 that

$$
\lim _{t \rightarrow \infty} \exp (t Q) f=\int f d m, \quad f \in H .
$$

This implies that as $t \rightarrow \infty v(t)$ tends in distribution to a normal random variable distributed by $m$. We shall use this fact in the next section.

In the sequel, we shall work in the Hilbert space $H$. This choice of space is canonical in the sense that if $\rho$ is any measure with the property that $Q$ is even only symmetric in $L^{2}(\rho)$, then $\rho$ must be a multiple of $m$.

In the theorem which follows, $\pi$ denotes the orthogonal projection onto 1 in $H$; i.e., $\pi g=\int g d m$ for any $g \in H$. Also, $D_{x}$ stands for $\partial / \partial x$, and all exponentials are given by their power series.

THEOREM 2.1. Let $f=\Sigma_{j \geqslant 0} f_{j} \epsilon^{j}$ be a formal power series in $\epsilon$ with coefficients which are $C^{\infty}$ in $x$ and assume that $\pi f=f_{0}$, i.e., $\pi f_{j}=0$ for $j \geqslant 1$. Then formal power series solutions $p=\Sigma_{j \geqslant 0} p_{j} \epsilon^{j}$ of the equation

$$
\frac{\partial p}{\partial t}=v \frac{\partial p}{\partial x}+\frac{1}{\epsilon} Q p, \quad \lim _{t \downarrow 0} p=f
$$

are unique, subject to the requirement $p_{j}(t, x, \cdot) \in H$. The function $f$ gives rise to such a solution if and only if

$$
f=\exp \left(\epsilon v D_{x}-\epsilon^{2} D_{x}^{2} / 4\right) f_{0} .
$$

Given an $f$ satisfying (2.5), we have that

$$
p=\exp \left(\epsilon v D_{x}-\epsilon^{2} D_{x}^{2} / 4+\epsilon t D_{x}^{2} / 2\right) f_{0} .
$$

Concerning convergence, we have that if $f_{0}$ is an entire function of $x \cdot$ of finite but arbitrary exponential type, then $f$ and $p$ converge for any $\epsilon>0$ to entire functions of the same exponential type. In this case, $p$ is a bonafide solution of (2.4). 
REMARKS. What makes the explicit formula (2.6) possible is the fortunate fact that the eigenvalue $\alpha(\lambda)$ of $Q-\lambda v$ which satisfies $\alpha(0)=0$ is $\alpha(\lambda)=$ $\lambda^{2} / 2$. In general, $\alpha(\lambda)$ is a power series in $\lambda$ with a finite radius of convergence. Formula (2.6) also reveals that power series solutions of (2.4) form a very restricted class. However, they provide a useful tool for proving limit theorems, as $\S 3$ will show.

PROof. We first show uniqueness for (2.4) by considering the equation

$$
\frac{\partial p}{\partial t}-v \frac{\partial p}{\partial x}=\frac{1}{\epsilon} \bar{Q} p, \quad \lim _{t \downarrow 0} p=f .
$$

Substituting the power series for $p$ into (2.7) and equating coefficients of powers of $\epsilon$, one obtains the equations

$$
\frac{\partial p_{j-1}}{\partial t}-v \frac{\partial p_{j-1}}{\partial x}=\bar{Q} p_{j}, \quad j \geqslant 0, p_{-1} \equiv 0 .
$$

Since $\lim _{t \downarrow 0} p_{j}=f_{j}$ and $\pi f_{j}=0, j \geqslant 1, f_{j}$ is determined by $p_{j-1}$ for $j \geqslant 1$. For $j=0,(2.8)$ yields $\bar{Q} p_{0}=0$. Since by Lemma 2.1 the null spaces of $Q$ and $\bar{Q}$ are both spanned by 1 , we conclude $. p_{0}(t, x, v)=p_{0}(t, x)$. For $j=1$, we obtain $\partial p_{0} / \partial t-v \partial p_{0} / \partial x=\bar{Q} p_{1}$. Since $\bar{Q}$ is selfadjoint and $(\bar{Q} f, f) \leqslant-(f, f)$, for all $f \in N(\bar{Q})^{\perp}$, this has a solution if and only if the left-hand side is perpendicular to 1 (see $\left[9\right.$, pp. 205-208]); i.e., $\pi \partial p_{0} / \partial t=\pi v \partial p_{0} / \partial x$ must hold.

But $\pi v \partial p_{0} / \partial x=\pi v \pi \partial p_{0} / \partial x \equiv 0$, and so $p_{0}=f_{0}$ follows. Since $v \in H$, we can continue in like fashion to determine $(1-\pi) p_{j}$ and $\pi p_{j}$ uniquely from the recursive equations

$$
\begin{gathered}
(1-\pi) \frac{\partial p_{j-1}}{\partial t}-(1-\pi) v \frac{\partial p_{j-1}}{\partial x}=\bar{Q}(1-\pi) p_{j},\left.\quad \pi p_{j}\right|_{t=0}=0 \\
\pi p_{j}=\int_{0}^{t} \pi v(1-\pi) \frac{\partial p_{j}}{\partial x}, \quad \text { for } j \geqslant 1 .
\end{gathered}
$$

Since $Q \subseteq \bar{Q}$, the uniqueness of formal power series solutions of (2.4) is shown.

This iterative procedure can be used to show the existence of formal power series solutions of (2.4). More directly, however, we see that $p$ given by (2.6) is a formal power series in $\epsilon$. Since

$$
\frac{\partial p}{\partial t}=\epsilon D_{x}^{2} p / 2, \quad Q p=\left(\epsilon^{2} D_{x}^{2} / 2-\epsilon v D_{x}\right) p,
$$

$p$ is also a formal solution of (2.4). Evaluating $p$ at $t=0^{+}$shows that $f$ must satisfy (2.5). Given such an $f$, we have from (2.3) that

$$
f=\sum_{n \geq 0} \frac{\epsilon^{n}}{n !} h_{n}(v)\left(D_{x} / 2\right)^{n} f_{0} .
$$


Since $\pi h_{n}=0$ for $n \geqslant 1$, it is clear that $\pi f=f_{0}$. On the other hand, if one starts from some $C^{\infty}$ function $f_{0}=f_{0}(x)$, then one follows exactly the proof given in [5, pp. 550-551] to show that the $f$ defined by (2.5) gives rise to one formal power series solution of (2.4).

The proof of convergence for any $\epsilon>0$ proceeds exactly as in [2, Theorem 4]. One makes use of the fact that the function of complex $z$ obtained by writing $z$ for $D_{x}$ in the exponential in (2.6) is an entire function of $z$.

Before we close this section, we indicate how formula (2.6) was discovered. Solving (2.9) recursively, one conjectures that

$$
\begin{gathered}
\pi p_{j}=\left(t^{j} / 2^{j} j !\right) D_{x}^{2 j} f_{0}, \quad j \geqslant 0, \\
\left.(1-\pi) p_{j}\right|_{t=0^{+}}=\left(1 / 2^{j} j !\right) h_{n}(v) D_{x}^{j} f_{0}, \quad j \geqslant 1 .
\end{gathered}
$$

We sum (2.10) over $j \geqslant 0$ and find

$$
\pi p=\exp \left(t \epsilon D_{x}^{2} / 2\right) f_{0} .
$$

Summing (2.11) over $j \geqslant 1$ and using (2.3), one sees that at $t=0^{+}$

$$
(1-\pi) p=\left(\exp \left(\epsilon v D_{x}-\epsilon^{2} D_{x}^{2} / 4\right)-1\right) \pi p .
$$

On physical grounds (see [2, Introduction]) one expects (2.13) to be true for all $t \geqslant 0$. Substituting (2.12) into (2.13), one arrives at (2.6).

3. Approximation of Brownian motion. We introduce some notation. Consider as in $\S 2$ the process $(x(t), v(t))$, where now $x(0)=0, v(0)=v$, and define for $f(x, v)$ bounded and smooth

$$
T_{t}^{\epsilon} f(x, v)=E_{(0, v)}\{f(x+\epsilon x(t / \epsilon), v(t / \epsilon))\}, \quad t \geqslant 0 .
$$

This is a solution of (2.4). For $f_{0}=f_{0}(x) \in C_{c}^{\infty}$, the space of $C^{\infty}$ functions with compact support, let $S_{t} f_{0}(x)=E f_{0}(x+w(t))$, where $w(t)$ denotes standard Brownian motion and $E$ the integral with respect to this process. We shall prove that for each $x$

$$
\lim _{\epsilon \downarrow 0}\left(T_{t / \epsilon}^{\epsilon} f_{0}\right)(x, v)=S_{t} f_{0}(x) \text {, uniformly for } v \text { in compacta. }
$$

We claim that (3.2) also yields the convergence of the $n$-dimensional distributions of $x^{\epsilon}(t) \equiv \epsilon x\left(t / \epsilon^{2}\right)$ to those of $w(t)$. This is equivalent to showing that the $n$-dimensional distributions of the increments of the $x^{\epsilon}(t)$ process converge to those of the increments of $w(t)$. Take, for example, $n=3$. Then for $f, h, s \in C_{c}^{\infty}$ and $0<t_{1}<t_{2}<t_{3}$, we have by the Markov property of $v(t)$ that

$$
\begin{aligned}
E_{(0, v)} & {\left[f\left(x^{\epsilon}\left(t_{1}\right)\right) \cdot h\left(x^{\epsilon}\left(t_{2}\right)-x^{\epsilon}\left(t_{1}\right)\right) \cdot s\left(x^{\epsilon}\left(t_{3}\right)-x^{\epsilon}\left(t_{2}\right)\right)\right] } \\
& =E_{(0, v)}\left[f\left(x^{\epsilon}\left(t_{1}\right)\right) \cdot E_{\left(0, v_{1}\right)}\left[h\left(x^{\epsilon}\left(t_{2}-t_{1}\right)\right) \cdot E_{\left(0, v_{2}\right)} s\left(x^{\epsilon}\left(t_{3}-t_{2}\right)\right)\right]\right],
\end{aligned}
$$


where $v_{1}=v\left(t_{1} / \epsilon^{2}\right)$ and $v_{2}=v\left(\left(t_{2}-t_{1}\right) / \epsilon^{2}\right)$. This can be shown to converge to

$$
\begin{aligned}
& E f\left(w\left(t_{1}\right)\right) \cdot E h\left(w\left(t_{2}-t_{1}\right)\right) \cdot E s\left(w\left(t_{3}-t_{2}\right)\right) \\
& \quad=E\left[f\left(w\left(t_{1}\right)\right) \cdot h\left(w\left(t_{2}\right)-w\left(t_{1}\right)\right) \cdot s\left(w\left(t_{3}\right)-w\left(t_{2}\right)\right)\right]
\end{aligned}
$$

by use of (3.2) and the fact that $v(t) \rightarrow N(0,1 / 2)$ in distribution as $t \rightarrow \infty$. Since one can prove that $E_{(0, v)}\left|x^{\epsilon}(t)\right|^{4} \leqslant C t^{2}, C$ independent of $\epsilon$, the weak convergence follows [8, pp. 33-35]. We shall prove (3.2) by considering $T_{t / \epsilon}^{\epsilon} f^{\epsilon}$, where $f^{\epsilon}$ is the power series in $\epsilon$ related to $f_{0}$ by (3.5). For another approach which exploits the Gaussian nature of the processes, see [6, pp. 60-61].

For $f_{0} \in C_{c}^{\infty}$ and $0<T<\infty$, we define $f_{0, T}$ by

$$
f_{0, T}(x)=\int_{|\gamma| \leqslant T} e^{i \gamma x} \hat{f}_{0} d \gamma,
$$

where $\hat{f}_{0}(\gamma)=(2 \pi)^{-1} \int e^{-i \gamma x} f_{0} d x$ is the Fourier transform of $f_{0}$. Now let

$$
f^{\epsilon}(x, v)=\int_{|\gamma| \leqslant T} e^{i \gamma x} \exp \left(\epsilon i \gamma v+\epsilon^{2} \gamma^{2} / 4\right) \hat{f}_{0} d \gamma .
$$

The function $f^{\epsilon}$ is entire in $x$ of finite type $T$, is bounded in $x$ and $v$, and is related to $f_{0, T}$ by (2.5). We denote by $p_{\epsilon}$ the convergent power series solution of (2.4) with initial data $f^{\epsilon}$, which can be written (see (2.6))

$$
p^{\epsilon}(t, x, v)=\int_{|\gamma|<T} e^{i \gamma x} \exp \left(\epsilon v i \gamma+\epsilon^{2} \gamma^{2} / 4-\epsilon t \gamma^{2} / 2\right) \hat{f}_{0} d \gamma .
$$

The key fact about $p^{\epsilon}$ is contained in the next lemma.

LEMMA 3.1. We have

$$
p^{\epsilon}=T_{t}^{\epsilon} f^{\epsilon} .
$$

REMARK. Although both functions in (3.3) solve (2.4) with initial data $f^{\epsilon}$, we do not know of any uniqueness results for this ultraparabolic equation.

Proof. We want to make use of the uniqueness part of Theorem 2.1. We first show that $T_{t}^{\epsilon} f^{\epsilon}$ is a power series in $\epsilon$. Let us write $g^{\epsilon}=\exp \left(-\epsilon^{2} D_{x}^{2} / 4\right) f_{0, T}$. We have from (2.5) and the entirety of $f_{0, T}$ that

$$
\begin{aligned}
T_{t}^{\epsilon} f^{\epsilon} & =E_{(0, v)}\left\{f^{\epsilon}(x+\epsilon x(t / \epsilon), v(t / \epsilon))\right\} \\
& =E_{(0, v)}\left\{\exp \left(\epsilon v(t / \epsilon) D_{x}\right) g^{\epsilon}(x+\epsilon x(t / \epsilon))\right\} \\
& =E_{(0, v)}\left\{\exp \left[\epsilon(x(t / \epsilon)+v(t / \epsilon)) D_{x}\right] g^{\epsilon}(x)\right\} .
\end{aligned}
$$

However, by adding the two equations in (2.1), we find that $x(t / \epsilon)+v(t / \epsilon)=$ 
$v+w(t / \epsilon)$. Thus,

$$
T_{t}^{\epsilon} f^{\epsilon}=E_{(0, v)}\left\{\exp \left[(\epsilon v+\sqrt{ } \epsilon w(t)) D_{x}\right] g^{\epsilon}(x)\right\}
$$

Since the odd moments of $w(t)$ vanish, this shows that $T_{t}^{\epsilon} f^{\epsilon}$ is a power series in $\epsilon$. Furthermore, (3.4) shows that as functions of $v$ the coefficients of the powers of $\epsilon$ in $T_{t}^{\epsilon} f^{\epsilon}$ are polynomials, which belong to $H$. Hence, (3.3) follows from the uniqueness part of Theorem 2.1.

Proof of (3.2). Fixing $x$, we have

$$
\begin{aligned}
\left|T_{t / \epsilon}^{\epsilon} f_{0}-S_{t} f_{0}\right| \leqslant & \left|T_{t / \epsilon}^{\epsilon} f_{0}-T_{t / \epsilon}^{\epsilon} f^{\epsilon}\right|+\left|T_{t / \epsilon}^{\epsilon} f^{\epsilon}-S_{t} f_{0}\right| \\
\leqslant & \left|T_{t / \epsilon}^{\epsilon} f_{0}-T_{t / \epsilon}^{\epsilon} f^{\epsilon}\right|+\int_{|\gamma|>T} \exp \left(-t \gamma^{2} / 2\right)\left|\hat{f}_{0}\right| d \gamma \\
& +\int_{|\gamma| \leqslant T} \exp \left(-t \gamma^{2} / 2\right)\left|\exp \left(i v \epsilon \gamma+\epsilon^{2} \gamma^{2} / 4\right)-1\right| \cdot\left|\hat{f}_{0}\right| d \gamma
\end{aligned}
$$

We use (3.3) to estimate the first term. The key idea below is to break up the $\xi$-integration first over $|\xi| \leqslant M$, then over $|\xi|>M$, for $M<\infty$. We have

$$
\begin{aligned}
& \left|T_{t / \epsilon}^{\epsilon} f_{0}-T_{t / \epsilon}^{\epsilon} f^{\epsilon}\right| \leqslant \iint\left|f_{0}(\eta)-f^{\epsilon}(\eta, \xi)\right| \cdot P_{(0, v)}\left\{\left(x+x^{\epsilon}(t)\right) \in d \eta, v\left(t / \epsilon^{2}\right) \in d \xi\right\} \\
& \quad \leqslant \sup _{|\xi| \leqslant M ;-\infty<x<\infty}\left|f_{0}(x)-f^{\epsilon}(x, \xi)\right|
\end{aligned}
$$

$$
\begin{aligned}
& +\sup _{-\infty<x, \xi<\infty}\left(\left|f_{0}\right|+\left|f^{\epsilon}\right|\right) \cdot P_{(0, v)}\left\{\left|v\left(t / \epsilon^{2}\right)\right|>M\right\} \\
& \leqslant \int_{|\gamma|>T}\left|\hat{f}_{0}\right| d \gamma+\sup _{|\xi|<M} \int_{|\gamma| \leqslant T}\left|f_{0}\right| \cdot\left|\exp \left(\epsilon \xi i \gamma+\epsilon^{2} \gamma^{2} / 4\right)-1\right| d \gamma \\
& +K \cdot P_{(0, v)}\left\{\left|v\left(t / \epsilon^{2}\right)\right|>M\right\} \quad(K \text { constant }) .
\end{aligned}
$$

However, since $v\left(t / \epsilon^{2}\right) \rightarrow N(0,1 / 2)$ as $\epsilon \downarrow 0$, the last term in (3.6) can be made small, uniformly for $v$ in compacta, by choosing $M$ large. Since choosing $T$ large, then $\epsilon$ small makes the other terms in (3.5) and (3.6) small uniformly for $v$ in compacta, the proof of (3.2) is complete.

4. Chapman-Enskog-Hilbert expansion for a general class of diffusion processes. Let $v(t)$ be the unique solution of the stochastic differential equation

$$
d v(t)=a(v(t)) d w+b(v(t)) d t, \quad v(0)=v \text { real, }
$$

where $a$ and $b$ are real-valued, Lipschitz continuous functions of $v, a$ is positive, and $a^{2}(v)+b^{2}(v) \leqslant K\left(1+v^{2}\right)$ for each $v$ ( $K$ a constant). The equation analogous to (2.4) takes the form

$$
\frac{\partial p}{\partial t}=v \frac{\partial p}{\partial x}+\frac{1}{\epsilon} Q p, \quad \lim _{t \downarrow 0} p=f .
$$


In (4.1),

$$
Q=1 / 2 a^{2}(v)\left(\partial^{2} / \partial v^{2}\right)+b(v)(\partial / \partial v)
$$

the generator of $v(t)$. For the purposes of the expansion, $f$ will be taken as a formal power series in $\epsilon$ with coefficients which are $C^{\infty}$ functions of $x$, and $a$ and $b$ will be assumed to be $C^{\infty}$ functions of $v$.

Let $m$ denote the locally finite measure whose density $r$ is given by

$$
r(v)=(a(v))^{-1} \exp \int_{0}^{v} \frac{b(u)}{a(u)} d u .
$$

We denote by $H$ the Hilbert space $L^{2}(m(d v))$ and by $D$ the set $\left\{g \in H: g \in C^{2}\right.$, $Q g \in H$. Since $Q$ can be written in the form

$$
Q=r^{-1} \frac{\partial}{\partial v}\left(\operatorname{ar} \frac{\partial}{\partial v}\right)
$$

it follows from [4, pp. 86, 189-190] that $Q$ on $D$ is essentially selfadjoint and the null spaces of $Q$ and its selfadjoint closure $\bar{Q}$ coincide.

We have the following result.

THEOREM 4.2. Assume the following three conditions:

(1) the nullspace $N(Q)$ of $Q$ consists of constants;

(2) 0 is an isolated point of the spectrum of $Q$;

(3) $v \in H$.

Then formal power series solutions $p=\Sigma_{j \geqslant 0} p_{j} \epsilon^{j}$ of (4.1) satisfying $p_{j} \in H$ as a function of $v$ are unique when they exist. Starting from any $C^{\infty}$ function $f_{0}=f_{0}(x)$, one can find a formal power series solution of (4.1), where $f$ is some formal power series involving $f_{0}$ and its derivatives and satisfying $\left.f\right|_{\epsilon=0}=f_{0}$.

Proof. We first show the existence part of the theorem. Substituting the formal power series for $p$ into (4.1) leads to the equations

$$
\frac{\partial p_{j-1}}{\partial t}-v \frac{\partial p_{j-1}}{\partial x}=Q p_{j}, \quad j \geqslant 0, \quad p_{-1} \equiv 0, \quad \lim _{t \downarrow 0} p_{0}=f_{0} .
$$

For $j=0$, we obtain $p_{0}(t, x, v) \equiv p_{0}(t, x)$. For $j \geqslant 1$, let us first consider the equations

$$
\frac{\partial p_{j-1}}{\partial t}-v \frac{\partial p_{j-1}}{\partial x}=\bar{Q} p_{j}, \quad j \geqslant 1, \lim _{t \downarrow 0} p_{0}=f_{0}
$$

and then show that the solutions of (4.4) actually solve (4.3). But by hypothesis (2), there is a $\delta>0$ such that $(\bar{Q} f, f) \leqslant-\delta(f, f)$, all $f \in N(\bar{Q})^{\perp}$. Since $\bar{Q}$ is selfadjoint, we know by hypotheses (1) and (3) that equations (4.4) can be 
solved recursively for $p_{j}$ in terms of $f_{0}$ and its derivatives. In solving (4.4), one finds that the functions $f_{j}=\lim _{t \downarrow 0} p_{j}$ are automatically determined in terms of $f_{0}$ and its derivatives.

We now show that the $p_{j}$ solve (4.3). This we do by first proving that $p_{j}$ is a weak solution of this equation; i.e., for any $\varphi=\varphi(v) \in C_{c}^{\infty}$, we have

$$
\int_{0}\left(Q^{+} \varphi\right) p_{j} d v=\int \varphi h_{j-1} d v,
$$

where $Q^{+}$is the formal adjoint of $Q$ and $h_{j-1}$ denotes the left-hand side of (4.3). Let us say (4.5) is shown. By induction, we assume $p_{j-1}$ is a $C^{\infty}$ function of $v$ (certainly true for $j=1$ ). But then $h_{j-1}$ is also, and since the coefficients of $Q$ are $C^{\infty}$, it follows by a corollary to a theorem of Friedrichs $[9, \mathrm{p}$. 178] that if (4.5) is true, then $p_{j}$, as a function of $v$, is a $C^{\infty}$ solution of $Q p_{j}=h_{j-1}$. To show (4.5), we define the $C_{c}^{\infty}$ function $\psi$ by $\psi=r^{-1} \varphi$ and argue as follows

$$
\begin{aligned}
\int \varphi h_{j-1} d v & =\int \psi\left(\bar{Q} p_{j}\right) r d v=\int(\bar{Q} \psi) p_{j} r d v \\
& =\int(Q \psi) p_{j} r d v=\int\left(Q^{+} \varphi\right) p_{j} d v .
\end{aligned}
$$

The second equality follows from the selfadjointness of $\bar{Q}$ in $H$, the third from the fact that $\psi \in \mathcal{D}$, and the last from the fact that $r Q=Q^{+} r$, which can be checked from (4.2). As the uniqueness part of the theorem should now be obvious, we are through.

Although we do not have in general the explicit formulae of $\S 2$, one can formally show the following facts when the three conditions of Theorem 4.2 hold. Assuming $m$ to be normalized to have total mass one and defining $x(t)=$ $\int_{0}^{t} v(s) d s$, we have, in the sense of weak convergence,

$$
\lim _{\epsilon \downarrow 0} \epsilon x(t / \epsilon)=\langle v\rangle t, \quad \lim _{\epsilon \downarrow 0} \epsilon x\left(t / \epsilon^{2}\right)-\langle v\rangle t \mid \epsilon=\sigma^{2} w(t),
$$

where $x(0)=0,\langle v\rangle=\int v m(d v)$ is the mean of $m$, and $\sigma^{2}=\int(v-\langle v\rangle)^{2} m(d v)$ is the variance of $m$. We omit the calculations.

Concerning the Chapman-Enskog-Hilbert development for multidimensional processes, let us take the case where the generator $Q$ of $v(t)$ is

$$
Q=\frac{1}{2} \sum_{1 \leqslant i \leqslant n} \frac{\partial^{2}}{\partial v_{i}^{2}}+\sum_{1 \leqslant i \leqslant n} b_{i}(\mathrm{v}) \frac{\partial}{\partial v_{i}} .
$$

It is known [7, p. 689], that a symmetrizing measure $m$ exists for such a $Q$ if and only if the vector field $\left(b_{1}(\mathrm{v}), \cdots, b_{n}(\mathrm{v})\right)$ is conservative, i.e., $\left(b_{1}(\mathrm{v}), \cdots\right.$, $\left.b_{n}(\mathrm{v})\right)=\operatorname{grad} u(\mathrm{v})$ for some $u$. In such a situation, the above goes through with little change. 


\section{BIBLIOGRAPHY}

1. R. Courant and D. Hilbert, Methoden der mathematischen Physik. Vol. I, Springer, Berlin, 1931; English transl., Interscience, New York, 1953. MR 16, 426.

2. R. Ellis, Chapman-Enskog-Hilbert expansion for a Markovian model of the Boltzmann equation, Comm. Pure Appl. Math. 26 (1973), 327-359.

3. Limit theorems for random evolutions with explicit error estimates, $Z$. Wahrscheinlichkeitstheorie und Verw. Gebiete 28 (1974), 249-256.

4. G. Hellwig, Differential operators of mathematical physics. An introduction, SpringerVerlag, Berlin and New York, 1964; English transl., Addison-Wesley, Reading, Mass., 1967. MR 29 \#2682; 35 \#2174.

5. H. P. McKean, Chapman-Enskog-Hilbert expansion for a class of solutions of the telegraph equation, J. Mathematical Phys. 8 (1967), 547-552. MR 35 \#1981.

6. E. Nelson, Dynamical theories of Brownian motion, Princeton Univ. Press, Princeton, N. J., 1967. MR 35 \#5001.

7. —, The adjoint Markov process, Duke Math. J. 25 (1958), 671-690. MR 21 \#365.

8. S. R. S. Varadhan, Stochastic processes, Notes based on a course given at New York University during the year 1967/68, Courant Inst. of Math. Sci., New York University, New York, 1968. MR 41 \#657.

9. K. Yosida, Functional analysis, 2nd ed., Die Grundlehren der math. Wissenschaften, Band 123, Academic Press, New York; Springer-Verlag, Berlin, 1968. MR 39 \#741.

DEPARTMENT OF MATHEMATICS, NORTHWESTERN UNIVERSITY, EVANSTON, ILLINOIS 60201 